\title{
COVID-19: opacification score is higher in the right lung and right lung involvement is a better predictor of ICU admission
}

To the Editor:

The global response to coronavirus disease 2019 (COVID-19) has resulted in a wealth of research. The accrual of data through electronic health records (EHR) facilitates the efficient interrogation of datasets. Indeed, many issues of relevance to the COVID-19 response have been explored in this way, the impact of ethnicity or angiotensin-converting enzyme inhibition on outcomes, to name but two $[1,2]$.

Large teaching hospitals in the capital were at the forefront of the COVID-19 outbreak in the UK, with over 1000 patients admitted in under 1 month. Research teams mobilised quickly to understand this new and unprecedented disease. We extracted data from our EHR to build a risk score that predicted critical care admission or death. The model included demographics, laboratory data and chest radiographic (CXR) severity [3].

The extent of CXR abnormality was scored using an adapted radiographic assessment of lung oedema for COVID-19, as proposed by Wong et al. [4]. The severity score attributes a number between 0 and 4 to each lung depending on extent of consolidation or ground glass opacification as follows: a score of 0 corresponding to no disease; 1 corresponding to $<25 \%$ extent; 2 to $25-49 \%$; 3 to $50-75 \%$; and a score of 4 corresponding to $>75 \%$ extent.

Admission CXRs on 1389 consecutive patients admitted with COVID-19 were evaluated. The first 200 radiographs were assessed by two independent scorers: there was high (90.5\%) inter-rater concordance. Subsequent review of between lung scores demonstrated moderate agreement $(r=0.72 ; \kappa=0.52)$.

A polychoric correlation comparing the degree of opacification by lung showed significant differences $(\mathrm{p}<0.0001)$. The striking differences were in the most severe categories. The right lung was more likely to be assigned the maximum extent score of $4: 11 \%$ versus $6 \%$ in the left lung. In addition, opacification of the right lung was a stronger predictor of admission to critical care or death (figure 1). This finding has not been reported previously or with other imaging modalities. We acknowledge important limitations in our work. We did not account for projectional image quality (e.g. anterior or posterior views). The scoring was done by acute physicians rather than radiologists.

The explanation for the apparent differential lung involvement in COVID-19 is unclear. If the finding is confirmed, it may offer insights into the pathobiology of COVID-19 in the lungs. The explanation may lie in anatomy: the right lung is anatomically larger than the left, with a larger main bronchus diameter and more segmental bronchi, possibly increasing viral delivery to respiratory epithelial surfaces. Conversely, it is also possible that the lung scoring is subject to perception bias with the cardiac silhouette distracting from left lung abnormalities. To our knowledge asymmetrical radiographic involvement in interstitial lung disease has not been previously reported.

As a research group with an interest in machine learning, it is interesting to reflect on the power of human observation. We look forward to this pattern being explored in other cohorts using tools such as volumetric computed tomography.

@ERSpublications

In COVID-19 the right lung has higher degree of opacification on plain radiograph than the left lung. Right lung opacificiation is a stronger predictor for critical care admission and death. https://bit.ly/ 36 dig $2 \mathrm{~N}$

Cite this article as: Nagra D, Russell M, Yates M, et al. COVID-19: opacification score is higher in the right lung and right lung involvement is a better predictor of ICU admission. Eur Respir J 2020; 56: 2002340 [https://doi.org/10.1183/13993003.02340-2020]. 


\begin{tabular}{|c|c|c|c|c|c|c|c|}
\hline a) & & & logic & ity sc & & & \\
\hline$\nexists$ & & 0 & 1 & 2 & 3 & 4 & Total \\
\hline$\stackrel{\bar{\nu}}{\partial}$ & 0 & 218 & 129 & 25 & 7 & 5 & 384 \\
\hline$\stackrel{\sim}{\sigma} \overrightarrow{0}$ & 1 & 100 & 227 & 79 & 31 & 11 & 448 \\
\hline 능 & 2 & 19 & 76 & 98 & 52 & 28 & 273 \\
\hline 으 & 3 & 7 & 21 & 36 & 78 & 53 & 195 \\
\hline$\ddot{\pi}$ & 4 & 3 & 4 & 8 & 20 & 54 & 89 \\
\hline & Total & 347 & 457 & 246 & 188 & 151 & 1386 \\
\hline
\end{tabular}

b)

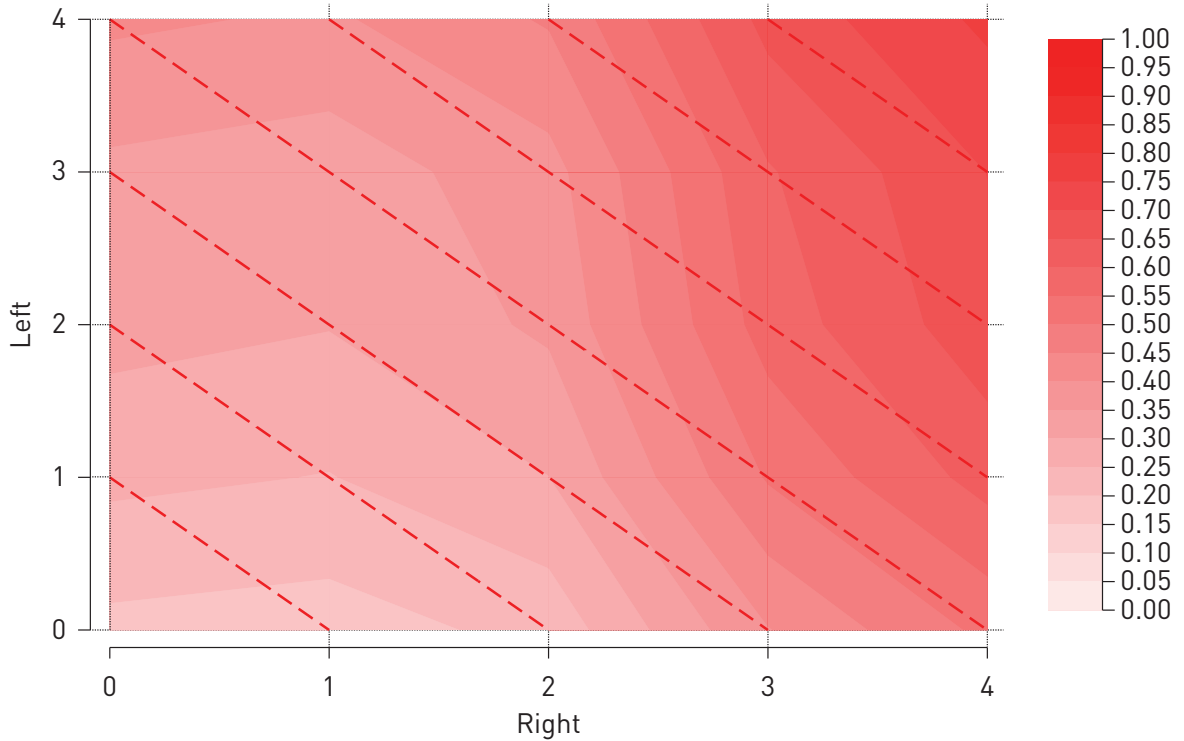

c)

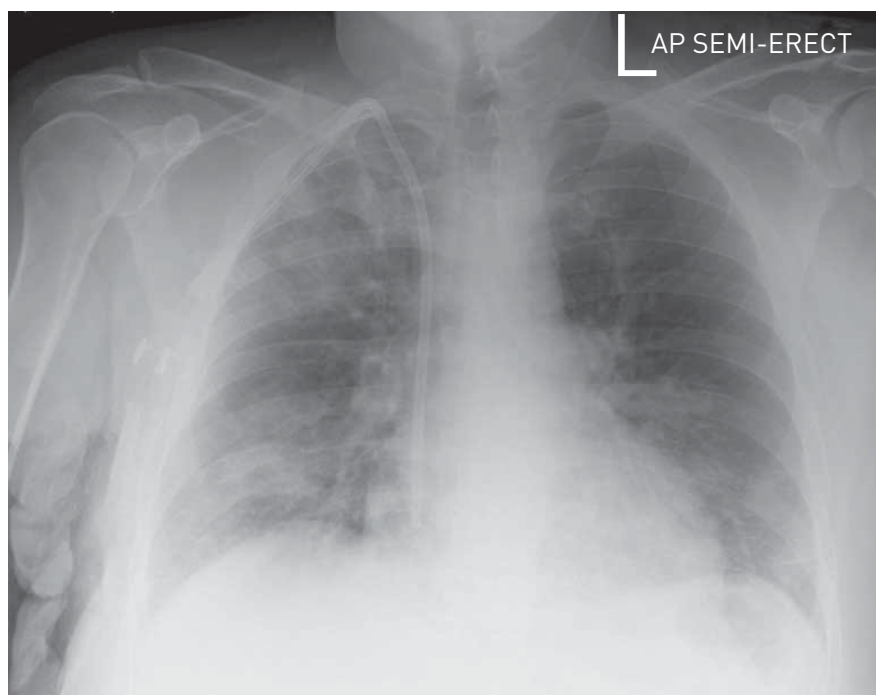

FIGURE 1 a) Radiological severity scores for left and right lung. b) A heat map of the predictive value of severity scoring for the left and right lung. Darker colour corresponds to a higher probability of a critical care admission or death ( $\mathrm{p}(\mathrm{TU} /$ death). c) An example chest radiograph showing greater opacification in the right lung field.

Deepak Nagra ${ }^{1}$, Mark Russell ${ }^{1}$, Mark Yates ${ }^{2}$, James Galloway ${ }^{2}$, Richard Barker ${ }^{3}$, Sujal R. Desai ${ }^{4}$ and Sam Norton ${ }^{5}$ ${ }^{1}$ King's College Hospital, London, UK. ${ }^{2}$ King's College London, Centre for Rheumatic Disease, London, UK. ${ }^{3}$ King's College Hospital, Respiratory Medicine, London, UK. ${ }^{4}$ The Royal Brompton and Harefield NHS Foundation Trust, Radiology, London, UK. ${ }^{5}$ King's College London, London, UK.

Correspondence: Deepak Nagra, King's College Hospital, Dept of Rheumatology, Portacabin B, London SE5 9RS, UK. E-mail: deepak.nagra@nhs.net 
Received: 15 June 2020 | Accepted after revision: 2 Sept 2020

Conflict of interest: None declared.

\section{References}

1 Williamson E, Walker AJ, Bhaskaran KJ, et al. OpenSAFELY: factors associated with COVID-19-related hospital death in the linked electronic health records of 17 million adult NHS patients. medRxiv 2020; preprint [https://doi. org/10.1101/2020.05.06.20092999].

2 de Abajo FJ, Rodríguez-Martín S, Lerma V, et al. Use of renin-angiotensin-aldosterone system inhibitors and risk of COVID-19 requiring admission to hospital: a case-population study. Lancet 2020; 395: 1705-1714.

3 Galloway JB, Norton S, Barker RD, et al. A clinical risk score to identify patients with COVID-19 at high risk of critical care admission or death: an observational cohort study. J Infect 2020; 81: 282-288.

4 Wong HYF, Lam HYS, Fong AHT, et al. Frequency and distribution of chest radiographic findings in COVID-19 positive patients. Radiology 2020; 296: E72-E78.

Copyright @eERS 2020.

This version is distributed under the terms of the Creative Commons Attribution Non-Commercial Licence 4.0. 